\title{
A New Perspective on Cyclic Loading Behavior Analysis of ATSP-Adjustable Telescopic Steel Prop S235GT Material Used in Structural Engineering
}

\begin{abstract}
Ö. KARAÇALI*
Department of Mechanical Engineering, Faculty of Engineering, Istanbul University, Avcılar, Istanbul, 34320, Turkey

In the fatigue life assessment of adjustable telescopic steel prop joints subjected to cyclic loading is critical for determining the number of cycles before fatigue failure of $235 \mathrm{GT}$ steel materials. The research aims in this reported work were to develop a computational material model and 3D finite-element analysis model to accurately predict the static and dynamic cyclic load-deformation characteristics of $235 \mathrm{GT}$ steel and adjustable telescopic steel prop components. Critical values for stress components, responsible for distinctive fatigue failure of the adjustable telescopic steel prop were obtained and the fatigue limits were illustrated in the Smith diagram. To achieve these, finite element analysis were employed to calculate compressive stresses and equivalent Von Mises stresses as well as the analysis by the strength of materials experimental procedure in laboratory conditions. Hence, through appropriate computer software, the fatigue strength of adjustable telescopic steel prop was automatically determined and expressed in the form of the Smith diagram. The results obtained by the finite-element analysis and calculations are entirely similar to that of strength of materials procedure. This research helps improving site safety to understand the current working load limit basis for the material design of adjustable telescopic steel prop structures and make this design risk-consistent to prevent fatal injuries in the building construction industry.
\end{abstract}

DOI: 10.12693/APhysPolA.129.436

PACS/topics: 81.70.-q, 81.70.Bt, 87.10.Kn

\section{Introduction}

Adjustable telescopic steel prop (ATSP) is the main structural members used for carrying loads of flooring and beams. Although this ATSP provides an ideal and economical method of supporting for all kind of frameworks, slabs, columns, beams and walls, there is no publication of the research problems and know-how presented until now in literature. Large number of obtainable adjustments between closed and open height, makes these ATSP the most versatile to use. Due to their extensive work heights and high carrying capacity, these components are under high compressive and tensile forces.

The research explained in this paper entails the cyclic loading and fatigue design of ATSP in agreement to the standard EN 1065 [1]. The carrying capacity is stated by the standard [2] as to the minimum value of the external load that is able to activate collapse mechanisms for flexural failure of the tubes. This investigation addresses the design requirements to consider the effects, on the stability of ATSP as well as flexural forces that affect the durability of ATSP. The fatigue issue of the ATSP is the condition whereby component material cracks or fails because of cyclic stresses applied below the ultimate strength of the material during flooring. Fatigue behavior was expressed through a Smith diagram of the critical stress of ATSP failure mode i.e. the Von Mises stresses that ensure their continuous endurance. Considering the

*e-mail: ozdogank@istanbul.edu.tr nonlinear coupling behavior between material, contact, and geometric effects were developed by three dimensional isoparametric finite element models of the ATSP joint. The elastoplastic solid elements with six hundred and seventy-eight nodes were employed for the effective three-dimensional finite-element modeling and analysis. A surface-to-surface contact algorithm in ANSYS [3] was used to simulate the interaction between contact surfaces. The static and dynamic cyclic loading problems solved with LS-DYNA [3], which was investigated in detail, was the ATSP as shown in Fig. 1. Detail of ATSP parts and research areas are also shown in Fig. 1. The design of the ATSP mechanical structures in this research consists of material selection, simulation and detailed cyclic loading analysis using finite element method (FEM) and Smith diagram followed by mechanical design, assembly, tests and integration as summarized in Fig. 1.

\section{Materials and method}

Finite element model of the intact ATSP was reconstructed from a normal industrial dataset. The model was reconstructed and rigorously examined for the mechanical responses to physiological floor loads. Since the whole region of the telescopic prop is of particular interest, each part was considered in the analysis. The main research objectives are: (1) to build a model for improving site safety to understand the current working load limit basis for the design of ATSP structures and make this design risk-consistent to prevent fatal injuries in the construction industry. (2) To compute strain and stress distribution on material, a real laboratory-testing environment and simulation model 


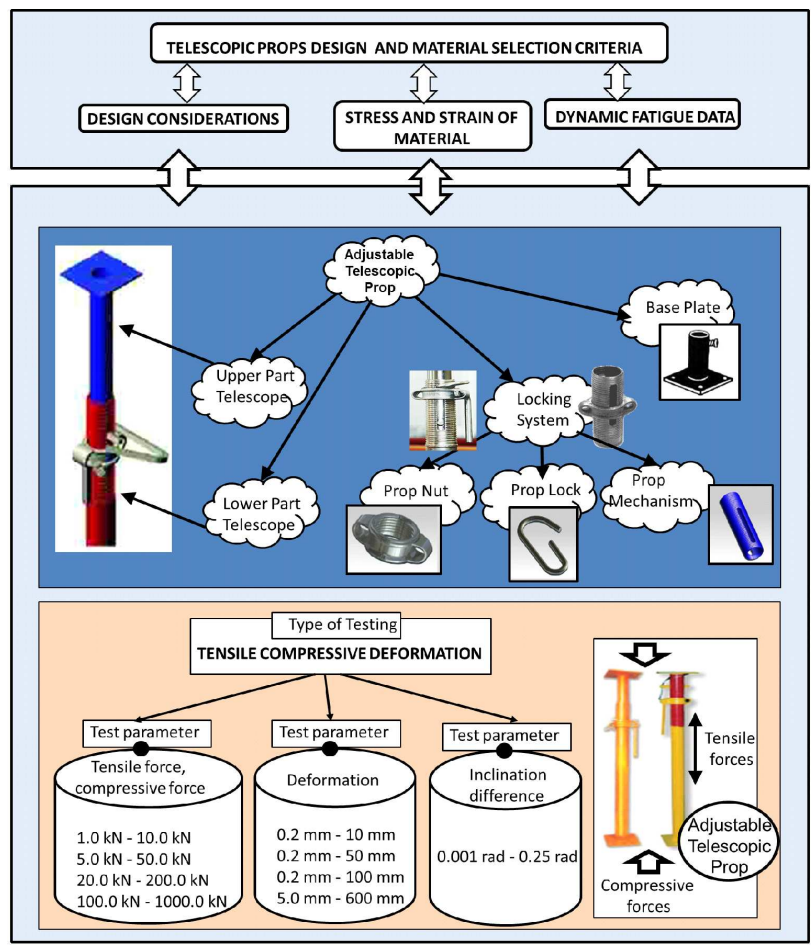

Fig. 1. Parts of adjustable telescopic prop-ATSP in various aspects of design considerations in the research.

were constructed including static and dynamic analysis. The testing was conducted according to DIN EN ISO/IEC 17025:2005 standards [4]. Joint test configurations for the experiments were conducted to investigate the stiffness and strength of ATSP joints in the vertical direction (rotation about the $z$-axis) and horizontal direction (rotation about the $y$-axis), see Fig. 1. The loading directions consist of up or down for bending about the $y$-axis. Mechanical testing of load bearing capacity was deformation behavior of ATSP as part of scaffold systems and scaffold structural elements of service, working scaffolds, falsework as well as formwork and racking systems. The ATSP has coarse adjustment with a pin inserted into holes in the inner tube and a means of fine adjustment using a threaded collar. The collar is provided with a swivel handle, which makes it easy to turn in confined spaces.

Static load of floor is considered to be the most critical load that could lead to fatigue problems of ATSP [5]. Equally it is a dynamic load with a large number of amplitude cycles, as the dead weight is a permanent load and contributes to the stress distribution significantly. The static analyses were based on the finite element analysis (FEA) models and aimed at calculating the deformations and stresses under operational conditions like static load. The deformations of the ATSP components were used to define the required maximum range of the segment position and supposed to correct the static misalignments by simulation. In addition the rigid body displacements and rotations of the ATSP components were calculated based on Von Mises equivalent stresses. This will be very useful for further design improvements and optimization studies. To be able to precisely predict the mechanical load behavior of material of ATSP, it is necessary to understand the physical phenomena to deduce the overall properties via FEM. Consequently, the development of quantitative displacement and stress distribution were presented by Von Mises method as shown in Fig. 2. Fairly high stresses occur in the framework structure under flooring gravity load on ATSP. The maximum Von Mises stresses in the order of $280 \mathrm{MPa}$ occur in a region below the segment supporting structure of ATSP. In order to meet the stress safety requirements several measures was taken in this research: (1) Reinforced the framework structure in the critical areas by increasing the cross-sectional areas of the beams, (2) modified the topology of the framework structure in the critical regions, (3) the yield strength limits of the steel materials used were $240 \mathrm{MPa}$ for S235GT and $327 \mathrm{MPa}$ for S355J2G3.

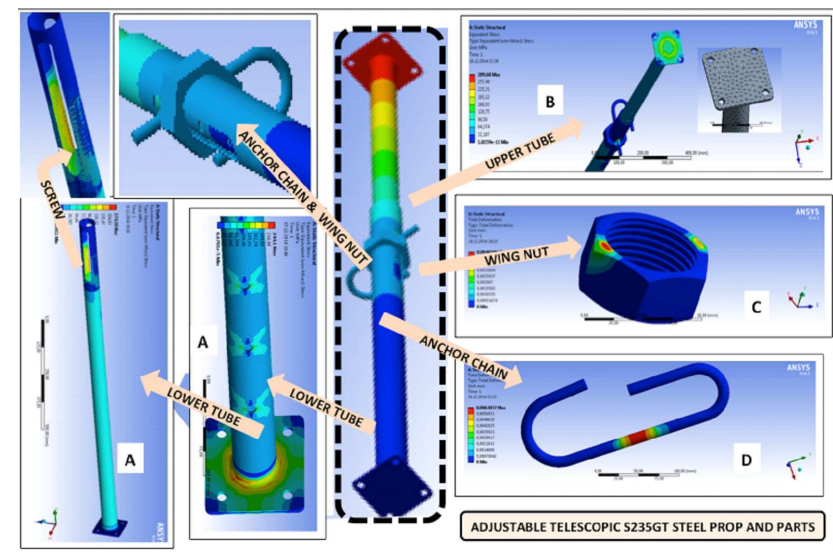

Fig. 2. Equivalent Von Mises stresses distributions on ATSP.

As a plane of symmetry existed along a section through the longitudinal axis of the ATSP, 3D finite element models developed using ANSYS software formed onehalf of the tube and its connection assembly as shown in Fig. 2. The analysis algorithm included nonlinearities caused by material, geometry, and contact with employing an energy-based convergence criterion - Von Mises method. The results obtained from the FEA for the loaddeflection areas were compared with those of the experiments, which showed that the FEA predicted the ATSP behavior closely with maximum errors of $1.5 \%$ for the steel S235GT. The accuracy of the FEA model was resulted from the exact values of the geometric parameters of the ATSP, which included dimensions and shapes of ATSP's end plate, length, cross-section, bolt head, bolt shank, and bolt nut. Important factor was calibrations for values for modulus of elasticity $E$, yield stiffness and equivalent Von Mises stresses in the model as shown in Fig. 2.

In this study, the Smith diagram for cyclic loading is a very useful fatigue analysis tool, presenting the areas, i.e. the stress levels at which the required fatigue life will 
not be reached. The corresponding deflection areas obtained, enable damage tolerant design, i.e. they divide the diagram area into two zones: the zone of stress states resulting in allowable and unallowable fatigue lives, that is in allowable and unallowable damage level as shown in Fig. 3. The basic scope of the dynamic analyses was to predict the dynamic behavior of the ATSP structure in the Smith diagram. The analysis aimed at calculating the regularities and geometric shapes belongs to ATSP material S235GT.

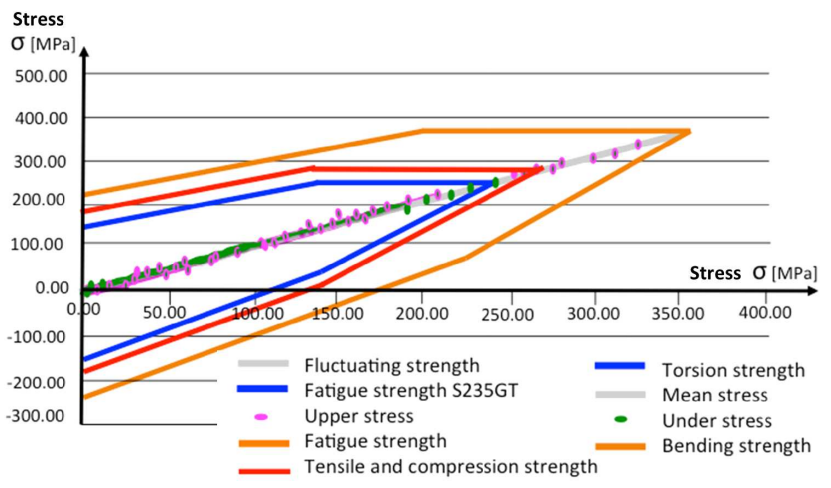

Fig. 3. Smith diagram under different load conditions for ATSP material-S235GT.

The scope of the analysis study presented in this section is to investigate the effect of the dynamic load contribution. The collapse of ATP structures due to excessive loading is an important safety aspect. Therefore the framework structure has to be designed such to withstand loadings that could cause torsion. Flexural loading failure might occur at loads smaller than that would be predicted from stress and strain calculations alone in a Smith diagram. Therefore, an extensive loading analysis study based on the models was performed by cyclic load of $N=10 \times 10^{6}$ number of cycles to failure in the laboratory testing conditions. By using the linear loading analysis, the theoretical strength of an ideal linear-elastic structure can be evaluated. Fluctuating strength occurs due to bending, tensile, compression, and torsion strength as shown in the Smith diagram in Fig. 3. The Smith diagram presented the stress states of ATSP in a timestress diagram; it is more appropriate to demonstrate the states of all stresses and its changes. The Smith diagram derived by the experimental-analytical method based on the loading tests and their FEM simulations were further validated in fatigue test. The mean Von Mises values and the corresponding amplitudes were inserted in the Smith diagram in Fig. 3. A comparison between the calculated and the permitted stresses contributed an explanation by Smith diagram for the fatigue behavior in the ATSP test. The Smith diagram was calculated to illustrate the mean and amplitude stress for each node in the structure with respect to the materials' endurance limit permissible stress alternating and pulsating strength. Figure 3 displays Smith diagram representing a uniform load case in $x$-direction with the component stress versus the mean stress. The pink and green dots are the lower and upper stress values. As can be seen the majority of the stresses lie very close to the diagonal line which represents the constant mean stress and are far away from the materials' limit strength curves in Fig. 3.

\section{Conclusions}

The exposed study, based on cyclic loading and computational finite element methodology, was applied to ATSP steel sample submitted to tensile, compression, and torsion strength test. The finite element analysis -ANSYS- based research and experimental work were conducted on S235GT steel material and adjustable telescopic steel props to identify their load-deflection behavior under the condition of static and dynamic loading and boundary conditions set from the adopted model. This approach in the research revealed that the maximum deflection occurred at the prop head and the maximum stresses concentrates at the interception of the telescopic prop with the load. The paper correspondingly presented the study of the Smith diagram as a tool for estimating both the stress states at the level of the compressiontensile and flexural loads in this research. The stress levels at which the required fatigue life was not reached within the unallowable damage level predicted from the Smith diagram in Fig. 3. Also, the initial geometric imperfections and material nonlinearity of all components of the ATSP system are incorporated in the models.

This investigation may aid improving site safety to understand the current working load limit basis for the design of ATSP' structures and make this design riskconsistent to prevent fatal injuries in the construction industry. Serious incidents may occur in involving the clamping of beams fitted on top of the ATS' leg making the flooring support. The results of systematic study are useful for cyclic load modeling of ATSP systems and the outcomes of this research may support to ATSP' designers and manufacturers to enterprise better and more safe flooring support products.

\section{Acknowledgments}

This research is funded by Istanbul University, Turkey, project No: 24082 (The Scientific Research Foundation - BAP). Such support is greatly acknowledged.

\section{References}

[1] A. Salvadori, J. Const. Steel. Res. 65, 1964 (2009).

[2] European Committee for standardization EN 1065, Adjustable telescopic steel props, product specifications, design and assessment by calculation and tests, 1999.

[3] ANSYS Manual, v. 16.5, ANSYS Inc., U.S.A., 2014.

[4] ISO/IEC 17025, General requirements for the competence of testing and calibration laboratories, 2005.

[5] A.S. Yassin, J.F. Martonik, Safety Sci. 42, 921 (2004). 